\title{
分析建国大学初级口语课教师对设计口试的题型
}

\section{THE PERCEPTION OF BINUS UNIVERSITY CHINESE SPEAKING TEACHERS TOWARDS THE SPEAKING TEST QUESTIONS}

\author{
Yetty（印尼建国大学中文系） \\ Chinese Department, Faculty of Language and Culture, Binus Universtiy \\ Jl. Kemanggisan Ilir III no.45, Kemanggisan/Palmerah, Jakarta Barat 11480
}

\section{内容提要}

\begin{abstract}
目前中文是世界上最重要的语言之一, 因为在世界上有六分之一的人使用中文为交际工具。 在印尼除了英语以外, 汉语现在也很普遍了。不少企业聘请职员的条件之一是必须会讲一口流 利的汉语。在学习一种语言中, 口语始终被作为学习语言的一个重要部分。在教学中, 口语课 是其中被对外汉语教学定为主干课程。教师为了使学生达到口语的好水平, 除了注意课堂上的 教学法以外, 也要设计标准的口试来评价学生们的口语表达能力。笔者想通过标准口试题型的 理论与对三位建国大学中文系的教师采访结果来分析建国大学中文系的口试题型与内容。笔者 所得到的结论是口试的主要题型要包括朗读、问答和讲述, 这些部分最能评到学生们的表达能 力、交际能力与语音能力的水平。关于口试内容三位教师认为大部分内容是要已经学过的, 小 部分可以从课本外的一些资料。
\end{abstract}

关键词: 汉语口语课，口试题型

\begin{abstract}
In relation to teaching and learning language, speaking always becomes an important skill. Speaking lesson has been designed as the main course in foreign language teaching and teachers have to master the teaching method in class, and he must be able to design a standard speaking test to assess student capability in speaking. In this article, the writer uses the theory of standard speaking test design, and combines it with the result of interview with three speaking teachers of the Chinese Department, BINUS University. This is to analyze the speaking test design and content. The result of the analysis shows that the important parts in speaking test design are reading, questions and answers, and the narration. It is used to assess student's expression, communication, and the level of phonetic abilities For the test content, the three teachers think that the material must be studied before and it is also possible to use small part from other materials outside the textbook.
\end{abstract}

Keywords: Chinese speaking lesson, speaking test questions 


\section{前言}

目前，中国是世界上经济发展最快的国家之一。汉语已经成为联合国的官方语言之一。 现在在印尼, 汉语扮演很大的角色, 因为目前在印尼有很多中资台资企业, 所以汉语成为必须 掌握的语言之一。因此老老少少都开始学习汉语, 不只是印尼华侨, 连印尼原住民也开始学 习汉语了。

汉语口语课是一门培养学生在实际生活中运用汉语进行口头交际的能力的专项语言技能课。 在对外汉语分技能设课的教学模式中, 口语课是非常重要的一课型。

初级的口试目的是考查学生是否具备了初步的汉语交际能力。口试在课程考试中十分重要, 对教学起指挥棒的作用。口试也要按照教学大纲的要求进行, 对教材和课堂教学有很大的依赖 性, 考试内容跟教学内容相一致, 即学什么, 考什么。

者想通过采访与参考资料来分析建国大学初级口语课的教师对设计口试的题型是否已经按 照口试题型的标准。

\section{内容}

\section{标准的口试题型}

考试的口语课教学的重要组成部分, 它通过对学生一组口语行为的抽象测量, 对学生的 口语交际能力作出推论。简单地说, 口试就是让被试开口说话, 根据他说出的话, 我们来评价 他的口语表达能力。口试也是单项技能考试, 它全面考查须生的口头表达能力。主要是高速组 织语言内容的能力, 正确选词造句、组句成段的能力。恰当选取表达方式的能力和善于运用声 音技巧的能力等口头表达微技能。

口试的题型有很多种, 下列就是一些题型多数既可以在口试使用。

(一) 朗读

正确的语音、语调是口试的基础, 所以, 口试中常常有朗读这一项。朗读的材料一般 是成段的文章, 但对初学者, 也可以使用单词和单句。朗读材料应该选择容易上口的, 句子不能太长, 不要有冷僻的字。

\section{（二）复述}

复述的目的是培养听读之后记忆语言材料的能力和接受信息并把它转化为自己的话表 达出来的技能, 同时也有丰富和规范学生口语的作用。复述的资料, 一般可以录在磁 带上, 让被试听, 也可以印成书面的, 让被试听读。被试听完或读完后, 立刻复述材 料中的内容。复述的材料不要太长, 也不要太难, 一般应选择有情节线索的故事, 这 样，记忆的负担不会太重。

（三）问答

问答就是主试提问, 被试回答, 这是口试中最常用的题型。主试提出的问答题的设计 应注意几点：第一，问题涉及的话题一般应该是被试熟悉、有可说的，如日常生活、 工作、学习、爱好等。第二, 一个问答题是由一组问题构成的, 在一组问题中涉及几 
个关系不大的话题。第三，问题的广度和难度应该逐步展开，前边几个问题要简单， 被试容易回答。

还需要注意的是, 问答题不适于用录音的方式, 因为问答是在具体情景中的、现场的 交流，当被试对一个问题不感兴趣或没有回答上来时，主试要及时调整问题的顺序或 内容，而这在录音的情况下是无法做到的。

（四）看图说话

看图说话就是让被试叙述图画上的内容，图画就是题干。口试所用的图画可以是单幅 的, 也可以是连环画, 使用哪一种, 根据需要而定。单幅图画一般是描绘一个场景, 其中有环境、有人物、被试所要做的, 就是用口头形式把这个场景叙述出来。连环画 一般描绘的是一个事件, 这个事件有起因, 有发展, 有结果, 被试要口头描述的就是 这个事件。使用连环画通常以 4 幅左右为宜。

（五）讲述

讲述按内容可归为两类, 一类是介绍某种情况或某件事情, 题目根讲故事差不多, 如 介绍他（她）家庭或学校还是也可以让被试讲一件经历过的事情。另一类可称为讲解, 可以先给被试一些资料, 如让被试看一段广告或影片, 然后让他给我们介绍片中的内 容。

\section{（六） 角色扮演}

把角色扮演作为口试题目时, 应该多准备一些场景, 让被试在其中挑选熟悉的场景, 以扮演合适的角色。

把角色扮演作为口试题目时, 应该多准备一些场景, 让被试在其中挑选熟悉的场景, 以扮演适合的角色。另外, 在角色扮演中, 主试可以作为另一个角色, 配合被试完成 交际任务。

（七）讨论

对于程度较高的被试, 可以使用讨论题。讨论题涉及的内容可以比较广泛, 给被试留 出充分发挥的余地。讨论题应该成组地出。在发表自己的意见前, 被试可以准备以两 分钟，写一个简单的提纲。被试的发言长度以两三分钟为宜。

\section{建国大学中文初级口试题型的调查}

通过调查, 笔者得了建国大学从 2010 至 2011 的中文初级口试题型。口试题型主要部 有朗读句子、回答问题、讲述、复述课文和分组会话。

（一）朗读句子

朗读题一般有 5 至 10 题。教师主要以被试的发音与声调来评价朗读部分。对教师们 来说朗读部分除了评价被试的语音能力也评价被试的认字能力。

（二）回答问题

回答问题一般有 6 至 8 题的选项, 被试只需要回答两个问题。被试是通过抽签方式来 选两个问题。教师认为回答问题的部分是能评价被试的交际能力。

（三）讲述

讲述题跟回答问题是一样, 一般有 4 至 8 题, 被试通过抽签来选一个题目来讲述。讲 述题能评到被试的表达能力。一般被试已经讲述完了某题目, 教师会跟被试进行关于 题目的小对话。这也能评价被试的交际能力。 
（四）复述课文

复述题一般有两种, 第一看短文复述, 第二看会话复述。复述部分也通过抽签方法来 选题, 被试只需要复述一个问题。教师给他们 3 分钟的时间来看课文与复述。

（五） 分组会话

一组大概有 4 至 5 个人进行一个会话。考试之前教师先给 4 个题目给被试, 被试必须 准备好 4 个题目的会话。考试当天, 被试通过抽签来选一个题目来进行会话。这能评 价被试的交际还有表达能力也评价一组的默契与合作。

笔者认为建国大学的中文初级口试题型已经有了口试最基本有的部分。虽然不是所有的 标准题型都有, 可主要的是这 5 个部分已经能评到被试的口语能力。

\section{建国大学中文初级口语教师对口试的题型}

为了了解建国大学的中文口语教师对口语考试的题型, 笔者通过采访得了信息。目前在建 国大学中文系教初级口语的教师有三个人。

第一位教师是曾微丹老师, 她教了一年的口语课。本教师还没做过口试题。她认为口语课 的主要目的就是能提高学生的中文表达能力, 提高学生的语音和语调能力和提高学生的中文交 际能力。关于口试题的内容, 她认为大部分的考题要从所学过的资料, 可以补充一点儿从课本 外的资料。对她来说标准的口试题必有朗读、复述、问答、看图说话和讲述。因为教学对象还 是初级学生所以朗读部分比较适合读课文而不是读句子, 朗读课文至少有 50 至 100 个字。对 于复述的题目, 教师认为看课文或会话来复述比较适合学生们的水平。教师认为现在的口试题 型能达到口试题型的标准因为口试题能评到学生表达能力、交际能力与语音能力的水平。

第二位教师是叶秀荟老师, 她教了一年多的口语课。本教师还没做过口试题。本教师认 为口语课的主要目的就是提高学生的中文表达能力和提高学生的中文交际能力。她也认为她认 为大部分的考题要从所学过的资料, 可以补充一点儿从课本外的资料。对她来说标准的口试题 必有朗读、问答、讲述和扮演角色。朗读部分, 本教师跟曾老师的想法不一样, 她认为初级学 生比较适合朗读句子, 可也可以给学生读课文, 使他们感到有挑战, 这能巩固他们对认字给语 音能力的水平, 但是课文内容不可以太多大约 50 至 100 个字。复述的题目, 教师认为看课文 或会话来复述。教师认为现在的口试题型已达到口试题型的标准因为口试题能评到被试表达能 力、交际能力与语音能力的水平。

第三位教师是康冰冰老师, 她教了一年多的口语课。本教师已经做过口试题。她认为口 语课的主要目的就是能提高学生的中文表达能力。关于口试题的内容, 她认为大部分的考题要 从所学过的资料, 可以补充一点儿从课本外的资料。对她来说标准的口试题必有朗读, 复述和 问答题。朗读部分较好是读课文, 课文内容大概 150 至 200 个字。复述的题目, 教师认为看课 文或会话来复述。教师认为现在的口试题型已达到口试题型的标准因为口试题能评到被试表达 能力、交际能力与语音能力的水平。

结论：通過調查，筆者得的結論是口試題型至少應該有朗讀、問答題、複述與講述與分組會話。 這五個部分能評量學習者的發音正確、表達能力與交際能力。每學期的期中考試或期末考試都 使用這五個題型。朗讀題雖然教師們要使用課文為試題模式, 但是學校認為用句子朗讀對初級 學習者比較適合, 以初級學習者的水平比較好先朗讀句子。複述題, 三個教師都認為複述課文 或會話比較好比聽錄音來複述。口試的內容, 教師們都同意必須使用已經教過的內容為主, 從 
課本外的內容為輔。建國大學華語文系初級口語老師都認為現在的口試題型已經達到口試的標 準題型, 因为能评到被试的口语能力。

\section{参考资料}

《语言测验理论与实现》，张凯，北京语言大学出版社，2002 年 9 月第一版

《汉语听力说话教学法》，杨惠元，北京语言大学出版社，2002 年 4 月第 3 版

《汉语口语课教学法》，蔡整芗，北京语言大学出版社，2009年 4 月第一版

《汉语课堂教学技巧》, 崔永华/杨寄洲, 北京语言大学出版社, 2002 年 7 月第一版

《实用对外汉语教学法》, 徐子亮/吴仁甫, 北京大学出版社, 2005 年 7 月第一版

http://zh.wikipedia.org/wiki/\%E8\%AF\%AD\%E8\%A8\%80. 2012-6-13

http://zhidao.baidu.com/question/13723470.html. 2006-10-11

http://zhidao.baidu.com/question/35224516.html?fr=qrl\&cid=93\&index=1. 2007-9-10 